\title{
Vitamin Measurement
}

National Cancer Institute

\section{Source}

National Cancer Institute. Vitamin Measurement. NCI Thesaurus. Code C74803.

The determination of the type and amount of vitamins in a sample. 